\title{
Dark Energy and Its Interactions with Neutrinos
}

\author{
Xinmin Zhang \\ Institute of High Energy Physics, Chinese Academy of Sciences, P.O. Box 918-4, Beijing 100049, P. R. China
}

\begin{abstract}
In this talk I will firstly review on the current constraints on the equation of state of the dark energy from observational data, then present a new scenario of dark energy dubbed Quintom. The recent fits to the type Ia supernova data and the cosmic microwave background and so on in the literature find that the behavior of dark energy is to great extent in consistency with a cosmological constant, however the dynamical dark energy scenarios are generally not ruled out, and one class of models with an equation of state transiting from below -1 to above -1 as the redshift increases is mildly favored. The second part of the talk is on interacting dark energy. I will review briefly on the models of neutrino dark energy.
\end{abstract}

\section{INTRODUCTION}

The recent observational data from type Ia supernova (SNIa), cosmic microwave background (CMB) radiation and large scale structure (LSS) have provided strong evidences for a spatially flat and accelerated expanding universe at the present time. In the context of Friedmann-Robertson-Walker cosmology, this acceleration is attributed to the domination of a component, dubbed dark energy. The simplest candidate for dark energy seems to be a remnant small cosmological constant. However, many physicists are attracted by the idea that dark energy is due to a dynamical component, such as a canonical scalar field $Q[1]$, named Quintessence. In this paper I will show firstly that the recent fits to the SNIa, $\mathrm{CMB}$ data and so on in the literature find that the behavior of dark energy is to great extent in consistency with a cosmological constant, however the dynamical dark energy scenarios are generally not ruled out and in fact one class of models with an equation of state (EOS) transiting from below -1 to above $-1[2]$ as the redshift increases, Quintom is mildly favored $3,4,4,5]$.

The second part of this talk is on interacting dark energy. Being a dynamical component, the scalar field of dark energy is expected to interact with the ordinary matters. There are many discussions on the explicit couplings of Quintessence to baryons, dark matter and photons, however for most of the cases the couplings are strongly constrained. But still there are exceptions. In this paper I will review about the recent studies on the models of neutrino dark energy. The paper is organized as follows: in section II I will review briefly about the current constraints on the dark energy from observational data; in section III I will review on the Quintom scenario of dark energy and in section IV I will study the neutrino dark energy models. The section $\mathrm{V}$ is the discussion and the summary of this talk.

\section{CURRENT CONSTRAINTS ON THE EQUATION OF STATE OF THE DARK ENERGY}

The quantity which characterizes the properties of the dark energy models is the equation of state defined as the ratio of the pressure to the energy density: $w=P / \rho$. For instances in models of dark energy provided by the (true or false) vacuum energy the equation of state $w$ is a constant and equals to -1 ; in theories of Quintessence $w$ varies as a function of redshift, however no matter how it evolves $w$ is restricted to be larger than -1 ; in theories of Phantom [6] where the kinetic term has a negative sign $w$ is smaller than -1 and no matter how it evolves it will never cross over the cosmological constant boundary into the regime of Quintessence.

In a model independent way to constrain the dark energy, one usually considers some kind of parameterizations of the equation of state. For instance, one possibility is expanding the equation of state in the powers of the redshift. For small redshift one has

$$
w(z)=W_{0}+W_{1} z .
$$

Another type of parametrization was proposed by Ref. 7]:

$$
w(z)=W_{1}+W_{a} z /(1+z) .
$$

Using the "gold" set of 157 SNIa published by Riess et al. in [8] many papers in the literature have presented the analysis on the constraints on the equation of state of the dark energy. The results in general show that the best fitting model of dark energy is the one with an equation of state transiting from below -1 to above -1 as the redshift increases, however at $2 \sigma$ the cosmological constant fits well to the data 2, 3].

Both parameterizations above make good approximations to probe the behavior of dark energy around the present epoch, while the former model leads to poor parameterization at very large redshift. As one example, in Ref. [9] we have considered one type of parametrization, the oscillating Quintom

$$
w(z)=W_{0}+W_{1} \sin z .
$$

The oscillating Quintom in (3) differs from the model with a linearly parametrized equation of state in (1) at high redshift, however at low redshift coincides with it. Fig.1 shows the constraints on the parameters of oscillating Quintom from SNIa and CMB. For comparison in Fig.2 we show the corresponding constraints on the 


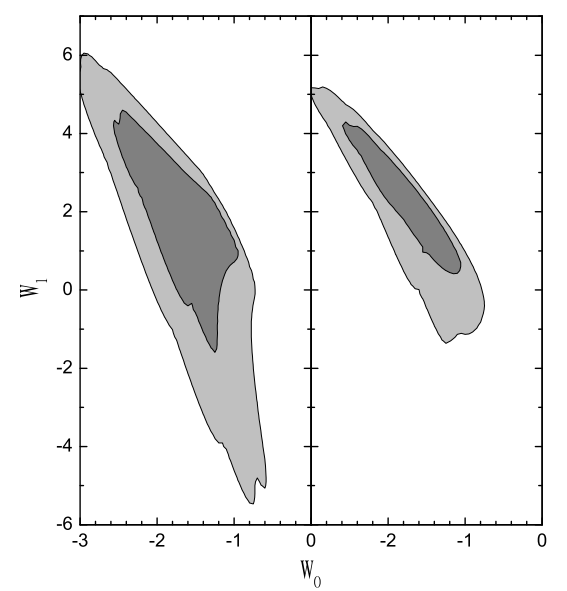

FIG. 1: SNIa and CMB constraints on the oscillating dark energy model 9]. The best fit values are shown in the centers of each panel. The grey and light grey areas show the $1 \sigma$ and $2 \sigma$ confidence regions respectively.

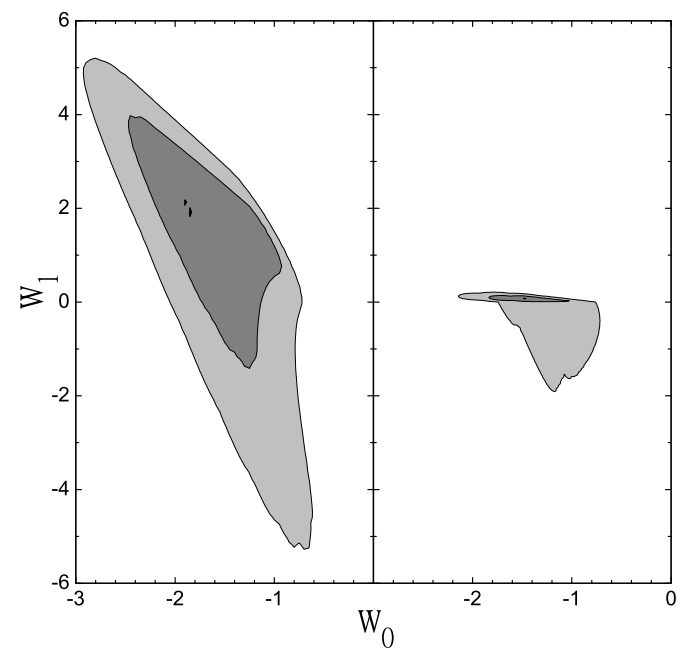

FIG. 2: SNIa and CMB constraints on the linear parametrized dark energy model[9]. Left : SNIa only; Right: SNIa + CMB constraints.

parameters $\left(W_{0}, \quad W_{1}\right)$ given in (1). One can see the similarity when considering only the SNIa data and the significant difference when including the CMB data. This exercise indicates the dependence of the "global fitting" results on the ways of parameterizing the equation of state of dark energy.

Another comment on the "global fitting" results is that the previous fittings in the literature have simply fully or partially neglected the perturbation of dark energy with an equation of state acrossing -1 . Recently with my colleagues I have developed a consistent way to include the perturbation of dark energy with equation of state crossing -1 and we have shown that in general the parameter space will get enlarged when including the perturbation than switching the dark energy perturbation off [10]. In Fig.3 we show the constraints on the parameters in Eq. (2) from SNIa, WMAP and SDSS and for comparison we plot in Fig.4 the corresponding constraints without the perturbation [1].

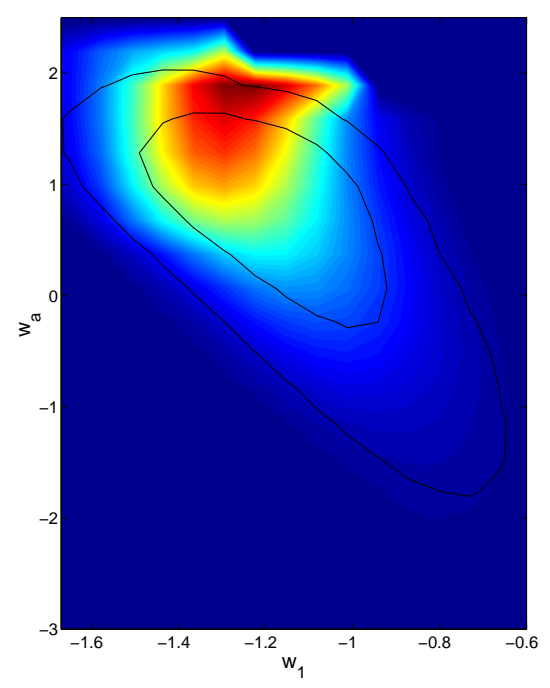

FIG. 3: Constraints on parameters of model (2) from SNIa + WMAP + SDSS with dark energy perturbation [1] .

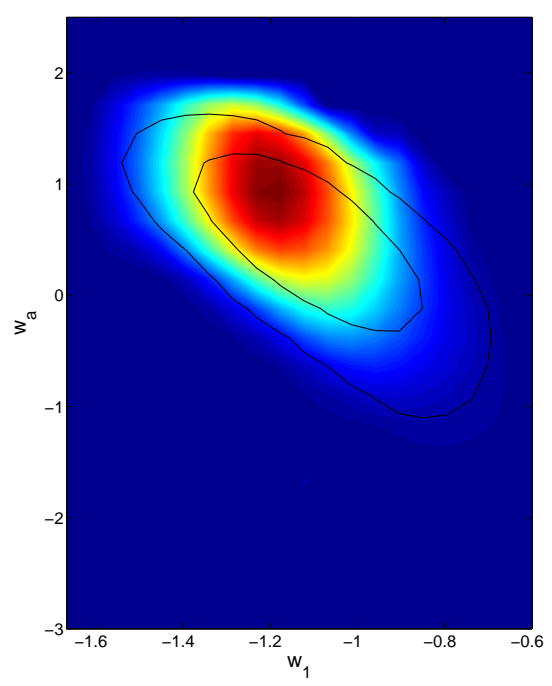

FIG. 4: Constraints on parameters of model (2) from SNIa + WMAP + SDSS without dark energy perturbation [1]]. 


\section{A NEW SCENARIO OF DARK ENERGY: THE QUINTOM}

Currently the cosmological constant fits well to the data, however observational data do not exclude the possibility of dynamical dark energy. Actually a class of models where the equation of state or an effective EOS evolves and crosses over the cosmological constant boundary is mildly favored. If such a result holds on with the accumulation of observational data, this would be a great challenge to current cosmology. Firstly, the cosmological constant as a candidate for dark energy will be excluded and dark energy must be dynamical. Secondly, the simple dynamical dark energy models considered vastly in the literature like the Quintessence, the Phantom or the K-essence 12 can not be satisfied either.

A simple Quintom model consists of two scalar fields, one being the Quintessence with the other being the Phantom field [3]. This type of Quintom model will provide a scenario where at early time the Quintessence dominates with $w>-1$ and lately the Phantom dominates with $w$ less than -1 , satisfying current observations. A detailed study on the cosmological evolution of this class of Quintom model is performed in Ref. $[5]$. The Quintom models are different from the Quintessence or Phantom in the determination of the evolution and fate of the universe. Generically speaking, the Phantom model has to be more fine tuned in the early epochs to serve as dark energy today, since its energy density increases with expansion of the universe. Meanwhile the Quintom model can also preserve the tracking behavior of Quintessence, where less fine tuning is needed.

In addition to the Quintom model mentioned above there are at least two more possibilities in the Quintom model buildings. One will be the scalar field models with non-minimal coupling to the gravity where the effective equation of the state can be arranged to change from above -1 to below -1 and vice versa. For a single scalar field coupled with gravity minimally, one may consider a model with a non-canonical kinetic term with the following effective Lagrangian 3 :

$$
\mathcal{L}=\frac{1}{2} f(T) \partial_{\mu} Q \partial^{\mu} Q-V(Q)
$$

where $f(T)$ in the front of the kinetic term is a dimensionless function of the temperature or scalar fields. During the evolution of the universe when $f(T)$ changes sign from positive to negative it gives rise to an realization of the interchanges between the Quintessence and the Phantom scenarios.

Since last year there have been a lot of studies in the literature on the Quintom-like model building ${ }^{1}$. In general as argued in Ref. 10 in the conventional case with an

\footnotetext{
${ }^{1}$ I apologize for not refereing to the papers relevant to this talk due to the limitation on the length of the paper.
}

single perfect fluid or a single scalar field one will not be able to realize a viable Quintom model, so to have the $w$ crossing -1 one needs to introduce extra degree of freedom. One possibility is to include multi fluids or multi scalar fields as shown above. Another possibility is to introduce the higher derivative operators to the lagrangian. This is the model proposed in Ref. [14] by introducing higher derivative operators to the Lagrangian. Specifically in [14] we considered a model with the Lagrangian

$$
\mathcal{L}=-\frac{1}{2} \nabla_{\mu} \phi \nabla^{\mu} \phi+\frac{c}{2 M^{2}} \square \phi \square \phi-V(\phi),
$$

where $\square \equiv \nabla_{\mu} \nabla^{\mu}$ is the d'Alembertian operator. The term related to the d'Alembertian operator is absent in the Quintessence, Phantom and the k-essence model, which is the key to make the model possible for $w$ to cross over -1 . We have proven in [14] this Lagrangian is equivalent to an effective two-field model

$$
\mathcal{L}=-\frac{1}{2} \nabla_{\mu} \psi \nabla^{\mu} \psi+\frac{1}{2} \nabla_{\mu} \chi \nabla^{\mu} \chi-V(\psi-\chi)-\frac{M^{2}}{2 c} \chi^{2},
$$

with the following definition

$$
\begin{aligned}
\chi & =\frac{c}{M^{2}} \square \phi, \\
\psi & =\phi+\chi .
\end{aligned}
$$

Note that the redefined fields $\psi$ and $\chi$ have opposite signs in their kinetic terms. One might be able to derive the higher derivative term in the effective Lagrangian (5) from fundamental theories. In fact it has been shown in the literature that this type of operator does appear as some quantum corrections or due to the non-local physics in the string theory. It is interesting and worthwhile to study further the implications of models with higher derivatives in cosmology (for a recent study see e.g. [15]).

In the following I summarize some of the interesting aspects associated with the Quintom scenario of dark energy:

1) Quintom dark energy gives rise to a new scenario of the evolution and the fate of the Universe. For example, in Ref. 13] we have studied a class of Quintom models with an oscillating equation of state and found that oscillating Quintom can unify the early inflation and current acceleration of the universe, leading to oscillations of the Hubble constant and a recurring universe. Our oscillating Quintom would not lead to a big crunch nor big rip. The scale factor keeps increasing from one period to another and leads naturally to a highly flat universe. The universe in this model recurs itself and we are only staying among one of the epochs, in which sense the coincidence problem is reconciled.

2) The study on the Quintom models provide us a way to include the perturbation of the dark energy consistently [10], which is important when performing the global fittings of the parametrized equation of state to the observational data including CMB, LSS and so on. As I 
pointed out in section II, neglecting the perturbation in general will lead to some bias in the fitting results.

3) The Quintom model might provide a way to solving the problem of the quantum instability inherited in the model of Phantom [16]. For Phantom theory, the equation of state is always less than -1 , however in the scenario of Quintom, $w<-1$ happens just for a short period of time very much like the "tachyon" existing only during the phase transition. It might be possible that the quantum instability problem be solved in the context of the higher derivative theory of Quintom model shown in (5). As pointed out in Ref. [17], the problem arises because $\chi$ and $\psi$ in Eq.(6) are quantized in canonical way independently. In fact, both of them are determined by $\phi$. A more appropriate quantization method seems to be possible to avoid the instability.

\section{NEUTRINO DARK ENERGY}

Recently there have been a lot of interests in the literature[18-35] in studying the possible connections between the neutrinos and the dark energy. There are at least two observations which motivate these studies: 1) the dark energy scale $\sim 10^{-3} \mathrm{eV}$ is smaller than the energy scales in particle physics, but interestingly is comparable to the neutrino masses; 2) in Quintessence-like models of dark energy $m_{Q} \sim 10^{-33} \mathrm{eV}$, which surprisingly is also connected to the neutrino masses via a seesaw formula $m_{Q} \sim m_{\nu}^{2} / M_{P l}$ with $M_{P l}$ the planck mass.

Are there really any connections between the neutrinos and dark energy? Given the arguments above it is quite interesting to make such a speculation on this connection. If yes, however in terms of the language of the particle physics it requires the existence of new dynamics and new interactions between the neutrinos and the dark energy sector.

Qualitatively these models have made at least two interesting predictions: 1) neutrino masses are not constant, but vary during the evolution of the universe; 2) $\mathrm{CPT}$ is violated in the neutrino sector due to the CPT violating Ether during the evolution of the Quintessence scalar field [19, 20]. Quantitatively these predictions will depend on the dynamics governing the coupled system of neutrino and dark energy. Here I focus on the models with mass varying neutrinos.

In general for the models of neutrino dark energy or interacting dark energy, the lagrangian can be written as

$$
\mathcal{L}=\mathcal{L}_{\nu}^{S M}+\mathcal{L}_{Q}+\mathcal{L}_{\text {int }},
$$

where $\mathcal{L}_{\nu}^{S M}$ is the lagrangian of the standard model (SM) describing the physics of the left-handed neutrinos, $\mathcal{L}_{Q}$ is for the dynamical scalar such as Quintessence. $\mathcal{L}_{\text {int }}$ in (9) is the sector which mediates the interaction between the dark energy scalar and the neutrinos.

At energy much below the electroweak scale, the relevant lagrangian for the neutrino dark energy is given by

$$
\mathcal{L}=\mathcal{L}_{\nu}+\mathcal{L}_{Q}+M(Q) \bar{\nu} \nu
$$

where $\mathcal{L}_{\nu}$ is the kinetic term of the neutrinos. The last term of Eq.(10) is the scalar field dependent mass of the neutrinos which characterizes the interaction between the neutrinos and the dark energy scalar. In the standard model of particle physics, the neutrino masses can be described by a dimension-5 operator

$$
L_{\not}=\frac{2}{f} l_{L} l_{L} H H+h . c,
$$

where $f$ is a scale of new physics beyond the Standard Model which generates the $B-L$ violations, $l_{L}, H$ are the left-handed lepton and Higgs doublets respectively. When the Higgs field gets a vacuum expectation value $<H>\sim v$, the left-handed neutrino receives a majorana mass $m_{\nu} \sim \frac{v^{2}}{f}$. In Ref. 21] we considered an interaction between the neutrinos and the Quintessence $Q$

$$
\beta \frac{Q}{M_{P l}} \frac{2}{f} l_{L} l_{L} H H+h . c,
$$

where $\beta$ is the coefficient which characterizes the strength of the Quintessence interacting with the neutrinos. In this scenario the neutrino masses vary during the evolution of the universe and we have shown that the neutrino mass limits imposed by the baryogenesis are modified.

The dim- 5 operator above is not renormalizable, which in principle can be generated by integrating out the heavy particles. For example, in the model of the minimal seesaw mechanism for the neutrino masses,

$$
L=h_{i j} \bar{l}_{L i} N_{R j} H+\frac{1}{2} M_{i j} \bar{N}_{R i}^{c} N_{R j}+\text { h.c. }
$$

where $M_{i j}$ is the mass matrix of the right-handed neutrinos and the Dirac masses of the neutrinos are given by $m_{D} \equiv h_{i j}<H>$. Integrating out the heavy righthanded neutrinos one will generate a dim-5 operator, however as pointed out in Ref. 21] to have the light neutrino masses varied there are various possibilities, such as by coupling the Quintessence field to either the Dirac masses or the majorana masses of the right-handed neutrinos or both.

In Ref. 23] we have specifically proposed a model of mass varying right-handed neutrinos. In this model the right-handed neutrino masses $M_{i}$ are assumed to be a function of the Quintessence scalar $M_{i}(Q)=\bar{M}_{i} e^{\beta \frac{Q}{M_{P l}}}$. Integrating out the right-handed neutrino will generate a dimension-5 operator, but for this case the light neutrino masses will vary in the following way

$$
e^{-\beta \frac{Q}{M_{P l}}} \frac{2}{f} l_{L} l_{L} H H+h . c .
$$

Some interesting features of the neutrino dark energy models can be summarized as follows: 
1) With mass varying the neutrinos become a part of the dark energy and play an different role in the determination of the evolution of the Universe than the traditional non-relativistic matter. In Ref. [25] we have studied in detail the cosmological evolution of the Universe in the scenario of mass varying neutrinos. We found that the neutrino density will not decrease and interestingly become the dominant one for a suitable choice of the model parameters. Mass varying neutrinos have interesting implications in leptogenesis 21, 23.

2) The predictions on the variation of the neutrino masses can be tested with Short Gamma Ray Burst [32], CMB and LSS [33] and much more interestingly and importantly in the experiments of neutrino oscillation 24, 28.

3) Neutrinos coupled to dark energy scalar 35] can provide a scenario of dark energy with the equation of state crossing the cosmological constant boundary of -1 . In a recent paper 34] we used the recently released SNIa data to constrain the couplings between the neutrinos and the dark energy scalar. We found the current data mildly favor the model where the Phantom-like scalar couples to the neutrinos.

\section{DISCUSSIONS AND CONCLUSION}

In this talk I have firstly given a brief review on the constraints on dark energy from observational data and presented a review on the Quintom model of dark energy. Secondly I have discussed the interacting dark energy and focused on the possible interactions between the dark energy and the neutrinos. If these interactions indeed exist, they will open up some possibilities of detecting the dark energy non-gravitationally. Before concluding I wish to add a new possibility to the list of interacting dark energy models [36] where the dark energy sector is closely connected to the Higgs and the Top quark in the standard model of elementary particle physics(SM). The motivation for this study is the following: the dark energy scale $\Lambda_{D E} \sim 10^{-3} \mathrm{eV}$ is numerically comparable to $\Lambda_{D E} \sim \frac{\Lambda_{F}^{2}}{M_{P l}}$ where $\Lambda_{F}$ is the Fermi scale, $M_{P l}$ the Planck mass. In the particle physics the sector of the electroweak symmetry breaking includes the Higgs boson. The top quark since its mass is heavier than all of the particles observed so far is believed to strongly couple to and play an essential role in understanding the physics associated to the Fermi scale. For example, in the models of top quark condensation, the Higgs is composite of the top pairs. Motivated by the observations above and numerically the masses of the top quark and the Higgs boson are order of the Fermi scale, the Quintessence scalar might preferably couple to the top quark and the Higgs than to other light particles. In this model the Higgs mass and the $\mathrm{CP}$ violating phase associated with the top Yukawa coupling vary during the evolution of the Quintessence. I will show that in this model the first order phase transition will be strong enough for electroweak baryogenesis without conflicting the current experimental limit on the Higgs mass.

In the SM, the Higgs potential at the tree level is given by

$$
V(H)=\lambda\left[H^{+} H-\frac{v^{2}}{2}\right]^{2},
$$

where the $\lambda$ is the self coupling constant of the Higgs field. When the $S U(2)$ doublet Higgs fields $H$ gets a vacuum expectation value $v$, the physical Higgs boson $h$ receives a mass $m_{h}=2 \lambda v^{2}$. Now we assume the interaction between the Higgs field $H$ and the Quintessence scalar $Q$ explicitly to be

$$
\beta \lambda \frac{\left(Q-Q_{0}\right)}{M_{P l}}\left[H^{+} H-\frac{v^{2}}{2}\right]^{2},
$$

where $Q_{0}$ is the value of the Quintessence field at present time and the parameter $\beta$ characterizes the strength of this type of interaction. Combining (15) and (16) we obtain an effective self coupling of the Higgs field

$$
\lambda^{e f f}=\lambda\left[1+\beta \frac{\left(Q-Q_{0}\right)}{M_{P l}}\right] .
$$

From (17) one can see the Higgs mass $m_{h}^{2}=2 \lambda^{e f f} v^{2}$ is now a function of the Quintessence field. At present time $m_{h}^{2}=2 \lambda v^{2}$ which recovers the results in the literature. At early time of the Universe, however $Q$ differs from $Q_{0}$, consequently the Higgs mass will also differ from its present value. This opens a possibility of having a light Higgs during the electroweak phase transition without conflicting the experimental limit at present time $^{2}$. Quantitatively the amount of the changes in the Higgs mass depends on the evolution of the Quintessence field. For a specific discussion we consider a model of Quintessence with a inverse power-law potential $V(Q)=$ $V_{0} Q^{-\alpha}$ 37]. In Fig. 5 we plot the evolution of the Quintessence scalar as a function of $\ln a$. One can see that in the early time during the period of the radiation dominate or matter dominate the scalar field was almost frozen and the value of $Q$ is nearly a constant, then change to the present value very recently. In Fig. 6 we plot the Higgs mass as a function of the $\ln a$ from which one can see that with a Higgs mass well above the current experimental limit $m_{h}>115 \mathrm{GeV}$, at temperature around $100 \mathrm{GeV}$ it could be as light as $35 \mathrm{GeV}$ with $\beta \sim 5$.

Introducing the coupling between the Quintessence scalar and the top quark will cause the top Yukawa coupling running. In the SM the top quark Yukawa coupling is

$$
y_{t} \overline{\Psi_{L}} \tilde{H} t_{R}
$$

\footnotetext{
2 this possibility has been mentioned in Ref 21$]$.
} 


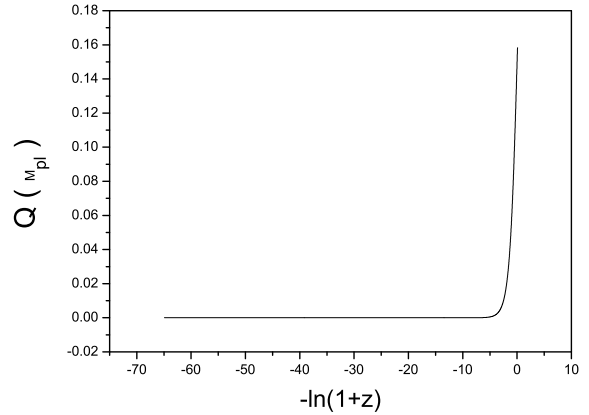

FIG. 5: Plot of the evolution of the scalar field of Quintessence with the inverse power-low potential $V(Q)=V_{0} Q^{-\alpha}$ where $\alpha=0.5$.

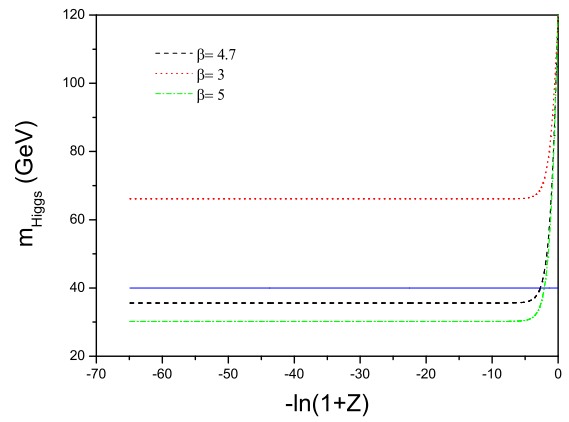

FIG. 6: Plot of Higgs mass with different value of parameter $\beta$ where $\beta=4.7$ (dashed line), $\beta=3$ (dotted line) and $\beta=5$ (dash dotted line); the horizontal line indicates the mass of the Higgs at $40 \mathrm{GeV}$.

where $\Psi_{L}=\left(t_{L}, b_{L}\right)$ is the $\mathrm{SU}(2)$ doublet quark of ${ }^{3}$ I thank Tim Tait and L. Fromme for discussions. the third generation. We introduce a coupling of the Quintessence to the term in (18)

$$
\delta \frac{\left(Q-Q_{0}\right)}{M_{P l}} \bar{\Psi}_{L} \tilde{H} t_{R}
$$

where $\delta$ is a parameter which characterizes this effective interaction. In general the Yukawa coupling in (18) and the coupling $\delta$ in (19) are complex with different phases. By the redefinition of the quark field and the convenience of the discussion we assume $y_{t}$ is real and $\delta$ has a phase. Introducing explicitly a phase $\xi$ and defining $\delta=c_{t} y_{t} e^{i \xi}$ we have an effective Yukawa coupling of the Top quark by combining (18) and (19)

$$
y_{t}^{e f f}=y_{t}\left[1+c_{t} e^{i \xi} \frac{\left(Q-Q_{0}\right)}{M_{P l}}\right] .
$$

One can see from the equation above that at present time the top quark mass $m_{t}=y_{t} v / \sqrt{2}$ is real, however at early time of the Universe it includes a complex CP violating phase. Unfortunately since the Quintessence field is almost a constant during the electroweak phase transition, the $\mathrm{CP}$ violation in the effective top Yukawa coupling will not help for the generation of the baryon number asymmetry ${ }^{3}$.

\section{ACKNOWLEDGEMENTS}

I would like to thank the organizers of PASCOS 2005 for invitation and giving me the opportunity to present this talk. I thank my collaborators for discussions. This work is supported in part by National Natural Science Foundation of China (grant Nos. 90303004, 19925523) and by Ministry of Science and Technology of China( under Grant No. NKBRSF G19990754).
[1] B. Ratra and P. J. E. Peebles, Phys. Rev. D37, 3406(1988); C. Wetterich, Nucl. Phys. B302, 668(1988); C. Wetterich, Astron. \& Astrophys. 301, 321(1995); J. A. Frieman, C. T. Hill, A. Stebbins, and I. Waga, Phys. Rev. Lett. 75, 2077(1995); I. Zlatev, L. Wang, and P. J. Steinhardt, Phys. Rev. Lett. 82, 896(1999); P. J. Steinhardt, L. Wang and I. Zlatev, Phys. Rev. D59, 123504(1999).

[2] e. g. U. Alam, V. Sahni, and A. A. Starobinsky, JCAP 0406, 008(2004); D. Huterer and A. Cooray, Phys. Rev. D71, 023506(2005); S. Nesseris and L. Perivolaropoulos, Phys. Rev. D70, 043531(2004); S. Hannestad and E. Mortsell, JCAP 0409, 001(2004); P. S. Corasaniti, M. Kunz, D. Parkinson, E. J. Copeland and B. A. Bassett, Phys. Rev. D70, 083006(2004); D. A. Dicus and W. W. Repko, Phys. Rev. D70, 083527(2004); B. A. Bassett, P. S. Corasaniti, and M. Kunz, Astrophys. J.617, L1 (2004); Y. G. Gong, Class. Quant. Grav.22, 2121(2005); Ch. Yeche et al., astro-ph/0507170
[3] B. Feng, X. Wang and X. Zhang, Phys. Lett. B607, 35 (2005).

[4] B. Feng, M. Li, Y. S. Piao and X. Zhang, astro-ph/0407432

[5] Z. Guo, Y. Piao, X. Zhang and Y. Zhang, Phys. Lett. B608, 177(2005).

[6] R. R. Caldwell, Phys. Lett. B545, 23(2002).

[7] M. Chevallier and D. Polarski, Int. J. Mod. Phys. D10, 213 (2001); E. Linder, Phys. Rev. Lett. 90, 091301(2003).

[8] A. G. Riess et al., Astrophys. J. 607, 665(2004).

[9] J. Xia, B. Feng and X. Zhang, Mod. Phys. Lett. A20, 2409(2005).

[10] G. Zhao, J. Xia, M. Li, B. Feng and X. Zhang, astro-ph/0507482

[11] J. Xia, G. Zhao, B. Feng, H. Li and X. Zhang (in preparation).

[12] A. Vikman, Phys. Rev. D71, 023515(2005).

[13] B. Feng, M. Li, Y. Piao and X. Zhang, astro-ph/0407432 
[14] M. Li, B. Feng, and X. Zhang, hep-ph/0503268

[15] A. Anisimov, E. Babichev and A. Vikman, J. Cosmol. Astropart. Phys. 0506, 006(2005).

[16] S. M. Carroll, M. Hoffman and M. Trodden, astro-ph/0301273 J. M. Cline, S. Jeon and G.D. Moore, hep-ph/0311312

[17] S. W. Hawking, Thomas Hertog, Phys.Rev.D65 103515(2002).

[18] P. Q. Hung, hep-ph/0010126 This paper considers the interaction between the Quintessence and the steril neutrinos.

[19] M. Li, X. Wang, B. Feng, X. Zhang, Phys. Rev. D65, 103511 (2002).

[20] M. Li, X. Zhang, Phys. Lett. B573, 20(2003).

[21] P. Gu, X. Wang and X. Zhang, Phys. Rev. D68, 087301(2003).

[22] R. Fardon, Ann E. Nelson and N. Weiner, JCAP 0410, 005(2004).

[23] P. Gu and X. Bi, Phys. Rev. D70, 063511(2004); X. Bi, P. Gu, X. Wang and X. Zhang, Phys. Rev. D69, 113007(2004).

[24] D. B. Kaplan, A. E. Nelson, N. Weiner, Phys. Rev. Lett. 93, 091801(2004).

[25] X. Bi, B. Feng, H. Li and X. Zhang, hep-ph/0412002

[26] P. Q. Hung and H. Pas, Mod. Phys. Lett. A20, 1209(2005).
[27] R. D. Peccei, Phys. Rev. D71, 023527(2005).

[28] V. Barger, P. Huber and D. Marfatia, hep-ph/0502196 M. Cirelli, M. C. Gonzalez-Garcia and C. Pena-Garay, Nucl. Phys. B719, 219(2005). V. Barger, D. Marfatia, K. Whisnant, hep-ph/0509163

[29] R. Horvat, astro-ph/0505507

[30] N. Afshordi, M. Zaldarriaga and K. Kohri, astro-ph/0506663

[31] R. Takahashi and M. Tanimoto, hep-ph/0507142.

[32] H. Li, Z. Dai and X. Zhang, Phys. Rev. D71, 113003(2005).

[33] A. W. Brookfield et al, astro-ph/0503349

[34] H. Li, B. Feng, J. Q. Xia and X. Zhang, astro-ph/0509272

[35] X. Zhang, H. Li, Y. Piao and X. Zhang, astro-ph/0501652

[36] "INTERACTING DARK ENERGY", Xinmin Zhang, Plenary talk at 12th International Conference on Supersymmetry and Unification of Fundamental Interactions (SUSY 04), Tsukuba, Japan, 17-23 Jun 2004. Published in ${ }^{*}$ Tsukuba 2004, Supersymmetry and unification of fundamental interactions* $73-85$ e-Print Archive: hep-ph/0410292

[37] B. Ratra and P. J. E. Peebles, Phys. Rev. D37, 3406(1988). 